\title{
Arithmetic Discrete Hyperspheres and Separatingness
}

\author{
Christophe Fiorio and Jean-Luc Toutant \\ LIRMM - CNRS UMR 5506 - Université de Montpellier II \\ 161 rue Ada - 34392 Montpellier Cedex 5, France \\ \{fiorio, toutant\}@lirmm.fr
}

\begin{abstract}
In the framework of the arithmetic discrete geometry, a discrete object is provided with its own analytical definition corresponding to a discretization scheme. It can thus be considered as the equivalent, in a discrete space, of a Euclidean object. Linear objects, namely lines and hyperplanes, have been widely studied under this assumption and are now deeply understood. This is not the case for discrete circles and hyperspheres for which no satisfactory definition exists. In the present paper, we try to fill this gap. Our main results are a general definition of discrete hyperspheres and the characterization of the $k$-minimal ones thanks to an arithmetic definition based on a non-constant thickness function. To reach such topological properties, we link adjacency and separatingness with norms.
\end{abstract}

\section{Introduction}

Discrete lines, namely the analogue of Euclidean lines in the discrete space $\mathbb{Z}^{2}$, have been widely studied. At first, J. Bresenham [1], H. Freeman [2] and A. Rosenfeld [3] have followed an algorithmic approach and have defined them as digitizations of Euclidean lines. They have provided tools for drawing and recognition [1, 2, 3. Later, J.-P. Reveillès has initiated the arithmetic discrete geometry [4] and has introduced the notion of arithmetic discrete line as solutions of a system of Diophantine inequalities, that is, as the subset of $\mathbb{Z}^{2}$ contained in a band. Such an approach enhances the knowledge of discrete lines. In addition to give new drawing [4] and recognition [5] algorithms, it directly links topological and geometrical properties of an arithmetic discrete line with its definition. For instance, its connectedness is entirely characterized by the width of the band, that is, its arithmetic thickness. In $d$-dimensional discrete spaces, the arithmetic discrete hyperplane [6] is a natural generalization of the arithmetic discrete line 4].

Similarly, first investigations into discrete circles have been algorithmic ones [7, 8, 9]. Discrete circles were only considered as digitizations of Euclidean circles. It is thus natural to ask whether or not J.-P. Reveillès' arithmetic approach is extendable to discrete circles and can supply them with an arithmetic definition. Such an extension has been proposed by É. Andres [10]. He 
has defined the discrete analytical hypersphere as solutions of a system of Diophantine inequalities, or in other words, as the subsets of $\mathbb{Z}^{d}$ contained in a ring of width its arithmetic thickness. Concentric discrete analytical hyperspheres tile the discrete space, but one does not retrieve topological properties as strict $k$-connectedness or $k$-minimality. Recently, an arithmetic definition implying a non-constant thickness function was proposed in [11. It provides discrete circles of integer parameters with 0-minimality or 1-minimality and gives an arithmetic characterization of the well-known Bresenham's circle [7].

In the present paper, we focus on discrete hyperspheres and on the arithmetic approach to generalize results of [11. We deeper study the notion of thickness. In particular, we characterize the $k$-minimal arithmetic discrete hypersphere by relating the thickness function to a particular norm, the $k$-minimality one, and to the local behaviour of the hypersphere.

The paper is organized as follows. First, we begin with some recalls on discrete geometry useful to fully understand the matter. Second, works already done on the discrete analytical hypersphere [10] and the arithmetic discrete circle [11] are presented. A general definition is then proposed to unify the both approaches. Next, we focus on the topological properties of $d$-dimensional discrete sets to characterize the $k$-minimal arithmetic discrete hypersphere.

\section{Basic Notions}

The aim of this section is to introduce the basic notions of discrete geometry used throughout the present paper. Let $d$ be an integer greater than 1 and let $\left\{\mathbf{e}_{\mathbf{1}}, \ldots, \mathbf{e}_{\mathbf{d}}\right\}$ denote the canonical basis of the Euclidean vector space $\mathbb{R}^{d}$. Let us call discrete set any subset of the discrete space $\mathbb{Z}^{d}$. The point $\mathbf{x}=\sum_{i=1}^{d} x_{i} \mathbf{e}_{\mathbf{i}} \in \mathbb{R}^{d}$, with $x_{i} \in \mathbb{R}$ for each $i \in\{1, \ldots, d\}$, is represented by $\left(x_{1}, \ldots, x_{d}\right)$. A point $\mathbf{v} \in \mathbb{Z}^{d}$ is a voxel in a $d$-dimensional space.

Definition 1 ( $k$-adjacency or $k$-neighborhood). Let $d$ be the dimension of the discrete space and $k \in \mathbb{N}$ such that $k<d$. Two voxels $\mathbf{v}=\left(v_{1}, \ldots, v_{d}\right)$ and $\mathbf{w}=\left(w_{1}, \ldots, w_{d}\right)$ are $k$-neighbors or $k$-adjacent if and only if:

$\|\mathbf{v}-\mathbf{w}\|_{\infty}=\max \left\{\left|v_{1}-w_{1}\right|, \ldots,\left|v_{d}-w_{d}\right|\right\}=1$ and $\|\mathbf{v}-\mathbf{w}\|_{1}=\sum_{i=1}^{d}\left|v_{i}-w_{i}\right| \leq d-k$.

Let $k \in\{0, \ldots, d-1\}$. A discrete set $\mathrm{E}$ is said to be $k$-connected if for each pair of voxels $(\mathbf{v}, \mathbf{w}) \in \mathrm{E}^{2}$, there exists a finite sequence of voxels $\left(\mathbf{s}_{\mathbf{1}}, \ldots, \mathbf{s}_{\mathbf{p}}\right) \in \mathrm{E}^{p}$ such that $\mathbf{v}=\mathbf{s}_{\mathbf{1}}, \mathbf{w}=\mathbf{s}_{\mathbf{p}}$ and the voxels $\mathbf{s}_{\mathbf{j}}$ and $\mathbf{s}_{\mathbf{j}+\mathbf{1}}$ are $k$-neighbors, for each $j \in\{1, \ldots, p-1\}$.

Let $\mathrm{E}$ be a discrete set, $\mathbf{v} \in \mathrm{E}$ and $k \in\{0, \ldots, d-1\}$. The $k$-connected component of $\mathbf{v}$ in $\mathrm{E}$ is the maximal $k$-connected subset of $\mathrm{E}$ (with respect to set inclusion) containing $\mathbf{v}$.

Definition 2 ( $k$-separating set). A discrete set $\mathrm{E}$ is $k$-separating in a discrete set $\mathrm{F}$ if its complement in $\mathrm{F}, \overline{\mathrm{E}}=\mathrm{F} \backslash \mathrm{E}$, has two distinct $k$-connected components. $\mathrm{E}$ is called a separator of $\mathrm{F}$. 
Definition 3 ( $k$-simple point, $k$-minimality). Let $d$ be the dimension of the space and $k \in \mathbb{N}$ such that $k<d$. Let also $\mathrm{F}$ and $\mathrm{E}$ be two discrete sets such that $\mathrm{E}$ is $k$-separating in $F$. A voxel $\mathbf{v} \in \mathrm{E}$ is said to be $k$-simple if $\mathrm{E} \backslash\{\mathbf{v}\}$ remains $k$-separating in $\mathrm{F}$. Moreover, a k-separating discrete set in $\mathrm{F}$ without $k$-simple points is said to be $k$-minimal in $\mathrm{F}$.

\section{Arithmetic Discrete Hyperspheres}

Initiated by J.-P. Reveillès with the arithmetic definition of the discrete line [4, the arithmetic discrete geometry has led to a wide literature, mainly about linear objects. Discrete lines [4], planes and hyperplanes [6] are now well characterized and only one parameter, the arithmetic thickness $\omega$, controls their topological properties. As far as we know, no satisfactory generalization to non-linear objects exists. É. Andres has defined the discrete analytical hypersphere [10] so as to obtain concentric hyperspheres tiling the space.

Definition 4 (Discrete analytical hypersphere [10]). Let $d$ be the dimension of the space. Let $r \in \mathbb{R}_{+}^{*}, \mathbf{o}=\left(o_{1}, \ldots, o_{d}\right) \in \mathbb{R}^{d}$ and $\omega \in \mathbb{R}_{+}^{*}$. The discrete analytical hypersphere $\mathbb{S}(\mathbf{o}, r, \omega)$ of center o, radius $r$ and arithmetic thickness $\omega$, is the subset of $\mathbb{Z}^{d}$ defined by:

$$
\mathbb{S}(\mathbf{o}, r, \omega)=\left\{\mathbf{v} \in \mathbb{Z}^{d} \mid\left(r-\frac{\omega}{2}\right)^{2} \leq \sum_{i=1}^{d}\left(v_{i}-o_{i}\right)^{2}<\left(r+\frac{\omega}{2}\right)^{2}\right\} .
$$

However, since no topological characterization is possible with this definition, the notion of arithmetic discrete circle was proposed in 11. Its analytical description is not based on the usual constant arithmetic thickness $\omega$, but on a thickness function $\omega: \mathbb{R}^{2} \longrightarrow \mathbb{R}_{+}^{*}$. The importance of keeping apart the analytical expression of the considered curve and the approximation induced by the discrete space, that is, the thickness, was also highlighted. Finally, those considerations lead to partial topological results on discrete circles. The naive and standard circles with integer parameters were characterized and Bresenham's circle [7] was provided with an arithmetic definition. For that, the thickness function is regarded as a measurement, by the usual discrete norms $\|\cdot\|_{1}$ and $\|\cdot\|_{\infty}$, of the local behaviour of the circle.

Definition 5 (Arithmetic discrete circle [11]). Let $\mathbf{o}=\left(o_{1}, o_{2}\right) \in \mathbb{R}^{2}, r \in$ $\mathbb{R}_{+}$. Let $\omega: \mathbb{R}^{2} \longrightarrow \mathbb{R}$ be a map. The arithmetic discrete circle $\mathrm{C}(\mathbf{o}, r, \omega)$ of center $\mathbf{o}$, radius $r$ and thickness function $\omega$ is the following set:

$$
\mathrm{C}(\mathbf{o}, r, \omega)=\left\{\mathbf{v} \in \mathbb{Z}^{2} \mid-\frac{\omega(\mathbf{v})}{2} \leq\left(v_{1}-o_{1}\right)^{2}+\left(v_{2}-o_{2}\right)^{2}-r^{2}<\frac{\omega(\mathbf{v})}{2}\right\} .
$$

In the present paper, we improve this last definition and generalize it to discrete hyperspheres, namely the $d$-dimensional case. 
Definition 6 (Arithmetic discrete hypersphere). Let $d$ be the dimension of the space, $r \in \mathbb{R}_{+}^{*}$ and $\mathbf{o}=\left(o_{1}, \ldots, o_{d}\right) \in \mathbb{R}^{d}$. Let $\omega_{1}: \mathbb{R}^{d} \longrightarrow \mathbb{R}_{-}$and $\omega_{2}: \mathbb{R}^{d} \longrightarrow \mathbb{R}_{+}$be maps. The arithmetic discrete hypersphere $\mathrm{S}\left(\mathbf{o}, r, \omega_{1}, \omega_{2}\right)$ with center $\mathbf{o}$, radius $r$ and thickness functions $\omega_{1}$ and $\omega_{2}$ is:

$$
\mathrm{S}\left(\mathbf{o}, r, \omega_{1}, \omega_{2}\right)=\left\{\mathbf{v} \in \mathbb{Z}^{d} \mid \omega_{1}(\mathbf{v}) \leq \sum_{i=1}^{d}\left(v_{i}-o_{i}\right)^{2}-r^{2}<\omega_{2}(\mathbf{v})\right\} .
$$

Contrary to Definition 5. Definition 6 includes two distinct thickness functions $\omega_{1}$ and $\omega_{2}$. That way, the global thickness can be distributed more or less inside or outside the hypersphere. This feature is interesting when one consider nonlinear analytical expression and when thickness should not be the same inside or outside the curvature.

Such a definition includes all previous attempts to define arithmetically discrete hyperspheres. In particular, the set of discrete analytical hyperspheres (see Definition [4) introduced by É. Andres [10] is included in the set of arithmetic discrete hyperspheres. They are defined using two thickness functions $\omega_{1}$ and $\omega_{2}$ such that $\omega_{1} \neq-\omega_{2}$.

Remark 1 (Discrete Analytical Hypersphere [10] and Arithmetic Discrete Hypersphere). The discrete analytical hypersphere $\mathbb{S}(\mathbf{o}, r, \omega)$ is the arithmetic discrete hypersphere $\mathrm{S}\left(\mathbf{o}, r, \omega_{1}, \omega_{2}\right)$ such that:

$$
\begin{aligned}
& \omega_{1}: \mathbb{R}^{d} \longrightarrow \mathbb{R}_{-} \\
& \mathbf{x} \longmapsto-r \omega+\frac{\omega^{2}}{4} \\
& \begin{aligned}
\omega_{2}: & \mathbb{R}^{d} \longrightarrow \mathbb{R}_{+} \\
\mathbf{x} & \longmapsto r \omega+\frac{\omega^{2}}{4} .
\end{aligned}
\end{aligned}
$$

In the same way, the arithmetic discrete circle introduced in [11] is an arithmetic discrete hypersphere.

Remark 2 (Arithmetic Discrete circles [11] and Arithmetic Discrete Hyperspheres). An arithmetic discrete circle $\mathrm{C}(\mathbf{o}, r, \omega)$ is an arithmetic discrete hypersphere $\mathbf{S}\left(\mathbf{o}, r, \omega_{1}, \omega_{2}\right)$ such that:

$$
\begin{aligned}
\omega_{1}: \mathbb{R}^{d} & \longrightarrow \mathbb{R}_{-} & \omega_{2}: \mathbb{R}^{d} & \longrightarrow \mathbb{R}_{+} \\
\mathbf{x} & \longmapsto-\frac{\omega(\mathbf{x})}{2} & \mathbf{x} & \longmapsto \frac{\omega(\mathbf{x})}{2} .
\end{aligned}
$$

Definition 6 allows to build a considerable amount of discrete objects and some do not look like what is expected from an hypersphere. Consequently, as in the case of the arithmetic discrete circle 11, suitable thickness functions are needed to characterize discrete hyperspheres with basic topological properties.

\section{Topology of $d$-Dimensional Discrete Sets}

Before defining suitable thickness functions, we have to determine which topological properties are meaningful for hyperspheres. For that, we focus on the 
more general case of $d$-dimensional discrete sets. The most studied one is the arithmetic discrete hyperplane [6] and the minimality (or the separatingness) is its best characterized topological property 6]. It seems anyway to be the most evident property for all $d$-dimensional discrete objects since it intuitively refers to the notion of objects without holes.

\section{1 $k$-Adjacency Norm}

The $k$-separatingness, and so the $k$-minimality, are reached when none of the voxels on one side of the separator is $k$-adjacent with a voxel on the other side. The definition of $k$-adjacency given in Section 2 is not easy to handle. Hence, we propose an equivalent and more formal expression. Indeed, two voxels are $k$ adjacent if they share at least $k$ coordinates and among the others, the greatest difference is equal to 1 .

Theorem 1. Let $d$ be the dimension of the space. Let $k \in \mathbb{N}$ such that $k<d$. Two voxels $\mathbf{v}=\left(v_{1}, \ldots, v_{d}\right)$ and $\mathbf{w}=\left(w_{1}, \ldots, w_{d}\right)$ are $k$-neighbor or $k$-adjacent if and only if:

$$
\left|v_{\sigma(d)}-w_{\sigma(d)}\right|+\sum_{i=1}^{k}\left|v_{\sigma(i)}-w_{\sigma(i)}\right|=1,
$$

with $\sigma$ the permutation of the set $\{1, \ldots, d\}$ such that, for all $i \in\{1, \ldots, d-1\}$, $\left|v_{\sigma(i)}-w_{\sigma(i)}\right| \leq\left|v_{\sigma(i+1)}-w_{\sigma(i+1)}\right|$.

Proof

$\mathbf{v}$ and $\mathbf{w}$ are $k$-adjacent. $\Leftrightarrow \max _{1 \leq i \leq d}\left\{\left|v_{i}-w_{i}\right|\right\}=1$ and $\sum_{i=1}^{d}\left|v_{i}-w_{i}\right| \leq d-k$.

$$
\begin{aligned}
& \Leftrightarrow\left|v_{\sigma(d)}-w_{\sigma(d)}\right|=1 \text { and } \sum_{i=1}^{k}\left|v_{\sigma(i)}-w_{\sigma(i)}\right|=0 . \\
& \Leftrightarrow\left|v_{\sigma(d)}-w_{\sigma(d)}\right|+\sum_{i=1}^{k}\left|v_{\sigma(i)}-w_{\sigma(i)}\right|=1 .
\end{aligned}
$$

In fact, the $k$-adjacency can be characterized by a norm that we call $k$-adjacency norm.

Proposition 1. Let $d$ be the dimension of the space and $k \in \mathbb{N}$ such that $k<d$. The map $[\cdot]_{k}$ defined by:

$$
\begin{aligned}
{[\cdot]_{k}: \mathbb{R}^{d} } & \longrightarrow \mathbb{R} \\
\mathbf{x} & \longmapsto\left|x_{\sigma(d)}\right|+\sum_{i=1}^{k}\left|x_{\sigma(i)}\right|
\end{aligned}
$$

with $\sigma$ the permutation of the set $\{1, \ldots, d\}$ such that, for all $i \in\{1, \ldots, d-1\}$, $\left|x_{\sigma(i)}\right| \leq\left|x_{\sigma(i+1)}\right|$, is a norm.

Proof. Here, positivity, scalability and triangle inequality are evident properties. 
Definition 7 ( $k$-adjacency norm). Let $d$ be the dimension of the space and $k \in \mathbb{N}$ such that $k<d$. We call $k$-adjacency norm, $[\cdot]_{k}$.

Notice that the $k$-adjacency norm is related to usual discrete norms $\|\cdot\|_{1}$ and $\|\cdot\|_{\infty}$. Let $d$ be the dimension of the space and $\mathbf{v} \in \mathbb{R}^{d}$, then $[\mathbf{v}]_{0}=\|\mathbf{v}\|_{\infty}$ and $[\mathbf{v}]_{(d-1)}=\|\mathbf{v}\|_{1}$.

\section{2 $(\lambda, k)$-Adjacency, $(\lambda, k)$-Separating Set and $(\lambda, k)$-Hull of a Voxel Set}

In Definition 6. we propose to distribute the thickness inside and outside the hypersphere. From a practical point of view, we can then consider the arithmetic discrete hypersphere as the union of two discrete hyperspheres, an outer one and an inner one. Since we are interesting in $k$-minimal discrete hyperspheres, inner and outer constituting hyperspheres can be thinner than $k$-minimal ones. So we need a notion more general than $k$-separatingness to define them. Since we characterize $k$-adjacency with a norm, we can extend it from the discrete space to the continuous one and define the $(\lambda, k)$-adjacency where distance between adjacent points can be a real number. It follows a generalization of the $k$-separating sets, the $(\lambda, k)$-separating sets. We thus control precisely the thickness of the set of voxels and can distribute the thickness as mentioned above.

Definition $8((\lambda, k)$-adjacency $)$. Let $d$ be the dimension of the space and $k \in$ $\mathbb{N}$ such that $k<d$. Let $\mathbf{x} \in \mathbb{R}^{d}$ and $\mathbf{y} \in \mathbb{R}^{d}$. The d-dimensional points $\mathbf{x}$ and $\mathbf{y}$ are $(\lambda, k)$-adjacent if and only if $[\mathbf{x}-\mathbf{y}]_{k} \leq \lambda$.

From $(\lambda, k)$-adjacency, the notion of $(\lambda, k)$-connected sets follows. It is thus natural to define $(\lambda, k)$-separating sets from $(\lambda, k)$-adjacency.

Definition 9 ( $(\lambda, k)$-separating set). A set $\mathrm{E}$ is $(\lambda, k)$-separating in a set $\mathrm{F}$ if its complements in $\mathrm{F}, \overline{\mathrm{E}}=\mathrm{F} \backslash \mathrm{E}$, has two distinct $(\lambda, k)$-connected components.

With Definition 9, we can distinguish two parts in a set of voxels $\mathrm{E} \subset \mathrm{U}$, its discrete $(\lambda, k)$-hull, which contains its voxels $(\lambda, k)$-adjacent with the exterior, and its $(\lambda, k)$-interior, $(\lambda, k)$-separated from the exterior.

Definition $10((\lambda, k)$-hull and $(\lambda, k)$-interior $)$. Let $d$ be the dimension of the space, $k \in \mathbb{N}$ such that $k<d$ and $\lambda \in \mathbb{R}$. Let also $\mathrm{O}$ be a set of voxels. The $(\lambda, k)$-hull, $\mathrm{H}_{(\lambda, \mathrm{k})}(\mathrm{O})$, of $\mathrm{O}$ based on the normal thickness $\lambda$ relatively to the $k$-adjacency norm $[\cdot]_{k}$ is defined as follows:

$$
\mathrm{H}_{(\lambda, \mathrm{k})}(\mathrm{O})=\left\{\mathbf{v} \in \mathrm{O} \mid \exists \mathbf{w} \in \mathbb{Z}^{d} \backslash \mathrm{O},[\mathbf{v}-\mathbf{w}]_{k} \leq \lambda\right\}
$$

The $(\lambda, k)$-interior of $\mathrm{O}$ is then $\mathrm{I}_{(\lambda, \mathrm{k})}(\mathrm{O})=\mathrm{O} \backslash \mathrm{H}_{(\lambda, \mathrm{k})}(\mathrm{O})$.

We focus on particular sets of voxels, the ones described by an analytical expression. So we now take care of the discrete hull of a discrete set defined in such a 
way. Let $f: \mathbb{R}^{d} \longrightarrow \mathbb{R}$ be a map. Then we define the discrete set $\mathrm{O}(f,+)$ such that:

$$
\mathrm{O}(f,+)=\left\{\mathbf{v} \in \mathbb{Z}^{d} \mid f(\mathbf{v}) \geq 0\right\} .
$$

Taking into account the map $f$, the $(\lambda, k)$-hull of the discrete set $\mathrm{O}(f,+)$ can be rewritten as follows:

$$
\mathrm{H}_{(\lambda, \mathrm{k})}(\mathrm{O}(f,+))=\left\{\mathbf{v} \in \mathbb{Z}^{d} \mid \exists \mathbf{x} \in \mathbb{R}^{d}, f(\mathbf{x})=0 \wedge f(\mathbf{v}) \geq 0 \wedge[\mathbf{v}-\mathbf{w}]_{k}<\lambda\right\} .
$$

According to Equation (7), a ( $\lambda, k)$-hull can be seen as a discrete object, based on a map $f,(\lambda, k)$-separating the discrete space. Unfortunately the set of voxels it contains is difficult to determine since the definition brings into play two different measurements, namely the norm $[\cdot]_{k}$ and the function $f$.

\section{$5 \quad(\lambda, k)$-Separating Discrete Hyperspheres}

The notion of $(\lambda, k)$-hull allows to define discrete $d$-dimensional hyperspheres of center $\mathbf{o}=\left(o_{1}, \ldots, o_{d}\right) \in \mathbb{Z}^{d}$ and radius $r \in \mathbb{R}_{+}^{*}$ with the particular map $s$ defined by:

$$
\begin{aligned}
s: \mathbb{R}^{d} & \longrightarrow \mathbb{R} \\
\quad \mathbf{x}=\left(x_{1}, \ldots, x_{d}\right) & \longmapsto \sum_{i=1}^{d}\left(x_{i}-o_{i}\right)^{2}-r^{2} .
\end{aligned}
$$

To go further, we consider the restriction of $s, s_{E}: E \longrightarrow \mathbb{R}$, to the subspace $E \subset \mathbb{R}^{d}$ such that:

$$
E=\left\{\mathbf{x}=\left(x_{1}, \ldots, x_{d}\right) \in \mathbb{R}^{d} \mid \forall i \in\{1, \ldots, d\}, x_{i}-o_{i} \geq 0\right\} .
$$

The $(\lambda, k)$-hull $\mathrm{H}_{(\lambda, \mathrm{k})}\left(\mathrm{O}\left(s_{E},+\right)\right)$ of the discrete set $\mathrm{O}\left(s_{E},+\right)$ in $E$ is then:

$$
\begin{aligned}
\mathrm{H}_{(\lambda, \mathrm{k})}\left(\mathrm{O}\left(s_{E},+\right)\right)=\left\{\mathbf{v} \in\left(\mathbb{Z}^{d} \cap E\right) \mid\right. & \exists x \in E, s_{E}(\mathbf{x})=0 \wedge s_{E}(\mathbf{v}) \geq 0 \\
& \left.\wedge[\mathbf{v}-\mathbf{w}]_{k}<\lambda\right\} .
\end{aligned}
$$

Since $s_{E}(\mathbf{x})=0$ and $s_{E}(\mathbf{v}) \geq 0$, we have the following equalities:

$$
\begin{aligned}
s_{E}(\mathbf{v}) & =\left|s_{E}(\mathbf{v})-s_{E}(\mathbf{x})\right|, \\
& =\left|\sum_{i=1}^{d} 2\left(v_{i}-o_{i}\right)\left(v_{i}-x_{i}\right)-\left(v_{i}-x_{i}\right)^{2}\right| .
\end{aligned}
$$

Since $\forall i \in\{1, \ldots, d\}, v_{i}-o_{i} \geq 0$, the absolute values are not required:

$$
s_{E}(\mathbf{v})=\sum_{i=1}^{d} 2\left(v_{i}-o_{i}\right)\left(v_{i}-x_{i}\right)-\left(v_{i}-x_{i}\right)^{2} .
$$

With the last condition on $\mathrm{H}_{(\lambda, \mathrm{k})}\left(\mathrm{O}\left(s_{E},+\right)\right),[\mathbf{v}-\mathbf{x}]_{k}<\lambda$, we give an upper bound on $s_{E}(\mathbf{v})$ and:

$$
\mathrm{H}_{(\lambda, \mathrm{k})}\left(\mathrm{O}\left(s_{E},+\right)\right)=\left\{\mathbf{v} \in\left(\mathbb{Z}^{d} \cap E\right) \mid 0 \leq s_{E}(\mathbf{v})<\sum_{i=d-k}^{d} 2 \lambda\left(v_{\sigma(i)}-o_{\sigma(i)}\right)-\lambda^{2}\right\},
$$


with $\sigma$ the permutation of the set $\{1, \ldots, d\}$ such that, for all $i \in\{1, \ldots, d-1\}$, $\left.\left.\mid v_{\sigma(i)}-o_{\sigma(i)}\right)|\leq| v_{\sigma(i+1)}-o_{\sigma(i+1)}\right) \mid$. Finally, thanks to the symmetries of $s$, we extend this last result to $\mathrm{H}_{(\lambda, k)}(\mathrm{O}(s,+))$ :

$$
\mathrm{H}_{(\lambda, k)}(\mathrm{O}(s,+))=\left\{\mathbf{v} \in \mathbb{Z}^{d}\left|0 \leq s(\mathbf{v})<\sum_{i=d-k}^{d}\right| 2 \lambda\left|v_{\sigma(i)}-o_{\sigma(i)}\right|-\lambda^{2} \mid\right\} .
$$

According to Definition [ and intrinsic separating properties, the discrete set $\mathrm{H}_{(\lambda, k)}(\mathrm{O}(s,+))$ is a good candidate for being an arithmetic discrete hypersphere. Before introducing such a definition, we propose notations to express the upper bound of the inequality in expression (8). Indeed, one can see it as a norm, depending on $k$, applied on a vector, depending on the local behaviour of $s$.

Proposition 2. Let $d$ be the dimension of the space and $k \in \mathbb{N}$ such that $k<d$. The map $] \cdot\left[_{k}\right.$ defined by:

$$
\begin{aligned}
] \cdot\left[{ }_{k}: \mathbb{R}^{d}\right. & \longrightarrow \mathbb{R} \\
\mathbf{x} & \longmapsto \sum_{i=d-k}^{d}\left|x_{\sigma(i)}\right|,
\end{aligned}
$$

with $\sigma$ the permutation of the set $\{1, \ldots, d\}$ such that, for all $i \in\{1, \ldots, d-1\}$, $\left|x_{\sigma(i)}\right| \leq\left|x_{\sigma(i+1)}\right|$, is a norm.

Proof. Here, positivity, scalability and triangle inequality are evident properties.

Definition 11 ( $k$-minimality norm). Let $d$ be the dimension of the space and $k \in \mathbb{N}$ such that $k<d$. We call $k$-minimality norm, the norm $] \cdot\left[{ }_{k}\right.$.

Similarly to the $k$-adjacency norm, the $k$-minimality norm is related to usual discrete norms $\|\cdot\|_{\infty}$ and $\|\cdot\|_{1}$. One has $] \mathbf{v}\left[_{(d-1)}=\|\mathbf{v}\|_{1}\right.$ and $] \mathbf{v}\left[_{0}=\|\mathbf{v}\|_{\infty}\right.$.

The upper bound in Equation (8) can be considered as the $k$-minimality norm of a particular vector. To achieve our goal to obtain a thickness depending on the local behaviour of $s$, and more generally of a function, we propose to define the Discrete Variation Map of a function, according to a thickness parameter:

Definition 12 (Discrete variation map). Let $d$ be the dimension of the space. Let $\lambda \in \mathbb{R}$ and $f: \mathbb{R}^{d} \longrightarrow \mathbb{R}$ be a function. The discrete variation map $\Delta_{\lambda} f$ of normal thickness $\lambda$ related to the function $f$ is:

$$
\begin{aligned}
& \Delta_{\lambda} f: \mathbb{Z}^{d} \longrightarrow \mathbb{R}^{d} \\
& \mathbf{v} \longmapsto\left(f\left(\mathbf{v}+\frac{\partial_{1} f(\mathbf{v})}{\left|\partial_{1} f(\mathbf{v})\right|} \lambda \mathbf{e}_{\mathbf{1}}\right)-f(\mathbf{v}), \ldots, f\left(\mathbf{v}+\frac{\partial_{d} f(\mathbf{v})}{\left|\partial_{d} f(\mathbf{v})\right|} \lambda \mathbf{e}_{\mathbf{d}}\right)-f(\mathbf{v})\right) .
\end{aligned}
$$

Now, we can define the $(\lambda, k)$-separating discrete hypersphere according to the Definition 6 of an arithmetic discrete hypersphere. 
Definition 13 ( $(\lambda, k)$-separating discrete hypersphere). Let $d$ be the $d i$ mension of the space and $k \in \mathbb{N}$ such that $k<d$. Let $\mathbf{o}=\left(o_{1}, \ldots, o_{d}\right)$ and $r \in \mathbb{R}_{+}^{*}$. Let $\lambda_{1} \in \mathbb{R}_{-}$and $\lambda_{2} \in \mathbb{R}_{+}$. The $(\lambda, k)$-separating discrete hypersphere $\mathrm{S}\left(\mathbf{o}, r, \omega_{\left(\lambda_{1}, k\right)}, \omega_{\left(\lambda_{2}, k\right)}\right)$ with center $\mathbf{o}$, radius $r$, normal thickness $\lambda=\lambda_{2}-\lambda_{1}$ and related to the k-minimality norm is defined by:

$$
\mathrm{S}\left(\mathbf{o}, r, \omega_{\left(\lambda_{1}, k\right)}, \omega_{\left(\lambda_{2}, k\right)}\right)=\left\{\mathbf{v} \in \mathbb{Z}^{d} \mid \omega_{\left(\lambda_{1}, k\right)}(\mathbf{v}) \leq s(\mathbf{v})<\omega_{\left(\lambda_{2}, k\right)}(\mathbf{v})\right\},
$$

with $\left.\omega_{\left(\lambda_{1}, k\right)}(\mathbf{v})=-\right] \Delta_{\lambda_{1}} s(\mathbf{v})\left[_{k}\right.$ and $\left.\omega_{\left(\lambda_{2}, k\right)}(\mathbf{v})=\right] \Delta_{\lambda_{2}} s(\mathbf{v})\left[_{k}\right.$.

Remark 3. In Definition 13. we arbitrarily decide to consider a large inequality outside the hypersphere and a strict one inside it. We also can do the opposite choice without changing the properties stated below.

Due to the underlying notion of $(\lambda, k)$-hull, the $(\lambda, k)$-separating discrete hypersphere has separating properties.

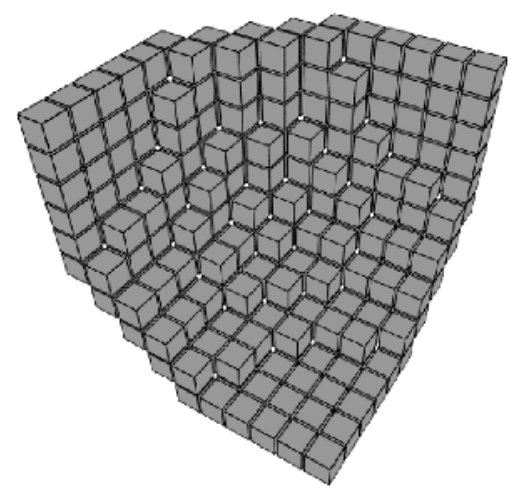

Fig. 1. Eighth of a 1-minimal discrete sphere with radius 9

Theorem 2 ( $k$-separating discrete hypersphere). The $(\lambda, k)$-separating discrete hypersphere $\mathrm{S}\left(\mathbf{o}, r, \omega_{\left(\lambda_{1}, k\right)}, \omega_{\left(\lambda_{2}, k\right)}\right)$ with normal thickness $\lambda=\lambda_{2}-\lambda_{1}$, such that $\lambda \in\left[1,+\infty\left[\right.\right.$, is $k$-separating in $\mathbb{Z}^{d}$.

Proof (Sketch). The $(\lambda, k)$-hull of a discrete object $(\lambda, k)$-separates its interior and the remaining discrete space. So the union of the $\left(\lambda_{1}, k\right)$-hull of an object and of the $\left(\lambda_{2}, k\right)$-hull of its complement is $\left(\lambda_{2}-\lambda_{1}, k\right)$-separating.

In particular, we characterize $k$-minimal arithmetic discrete hyperspheres. For instance, a part of a 1-minimal sphere is drawn in Figure1. We notice that voxels in one of the 2-connected components of the exterior are 0-adjacent with voxels of the other 2-connected component as expected.

Theorem 3 ( $k$-minimal discrete hypersphere). The $(\lambda, k)$-separating discrete hypersphere $\mathrm{S}\left(\mathbf{o}, r, 0, \omega_{(1, k)}\right)$ is $k$-minimal in $\mathbb{Z}^{d}$ for $k \in\{0, \ldots, d-1\}$. $(\lambda, k)$-separating discrete hyperspheres $\mathrm{S}\left(\mathbf{o}, r, \omega_{(\varepsilon-1, k)}, \omega_{(\varepsilon, k)}\right)$ are also k-minimal for $\varepsilon \in[0,1[$ and $k \in\{1, \ldots, d-1\}$. 
Proof (Sketch). In a discrete hypersphere $\mathrm{S}\left(\mathbf{o}, r, \omega_{(\varepsilon-1, k)}, \omega_{(\varepsilon, k)}\right)$, a $k$-simple point is a voxel which is just $(k-1)$-connected with one of both $k$-connected components of the exterior. Due to the symmetry of the hypersphere, if such a configuration does not appear in the neighborhood of the voxels $\mathbf{v}$ such that $\left|v_{1}-o_{1}\right| \simeq \cdots \simeq\left|v_{d}-o_{d}\right| \simeq(r / \sqrt{d})$, it then appears nowhere and the discrete hypersphere is $k$-minimal. Finally, we verify that $(1, k)$-separating discrete hyperspheres $\mathrm{S}\left(\mathbf{o}, r, 0, \omega_{(1, k)}\right)$ with $k \in\{0, \ldots, d-1\}$ and $\mathrm{S}\left(\mathbf{o}, r, \omega_{(\varepsilon-1, k)}, \omega_{(\varepsilon, k)}\right)$ with $k \in\{1, \ldots, d-1\}$ are $k$-minimal in $\mathbb{Z}^{d}$.

Now, we define naive and standard arithmetic discrete hyperspheres as already done for arithmetic discrete hyperplanes. In Figure 2(a), we notice, as expected, that some voxels in one side of the naive discrete hypersphere are 1-adjacent with voxels on the other side. On the contrary, the standard discrete sphere in Figure 2(b) does not have any hole.

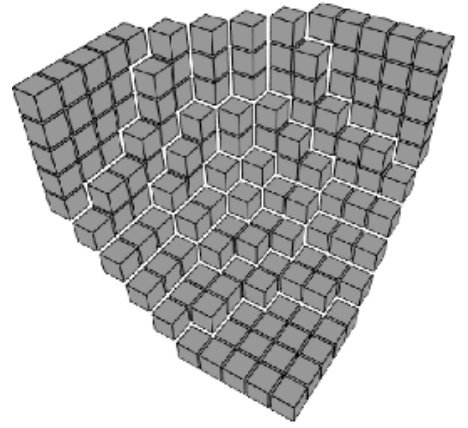

(a) Eighth of a naive discrete sphere

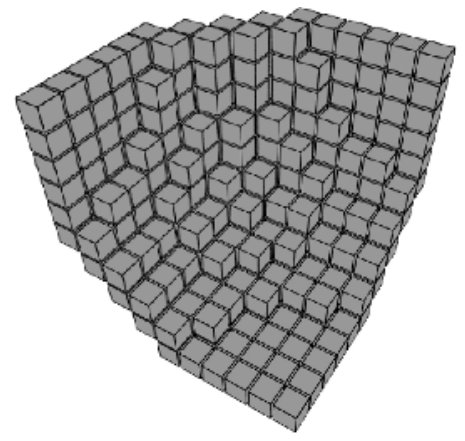

(b) Eighth of a standard discrete sphere

Fig. 2. Naive and standard discrete spheres with radius 9

Definition 14 (Naive and standard discrete hyperspheres). Let $d$ be the dimension of the space. A naive (respectively standard) discrete hypersphere is $a(d-1)$-minimal (respectively 0-minimal) one.

In the $d$-dimensional discrete space, the most studied object is the discrete hyperplane [6]. It is a generalization of the arithmetic discrete line 4]. Provided with a particular arithmetic thickness, the arithmetic discrete hyperplane presents basic topological properties, that is, the $k$-minimality [6]. Such a thickness can be defined by combining the $k$-minimality norm and the discrete variation map associated to the hyperplane. In Figure 3, $k$-minimal discrete planes are drawn to be compared with $k$-minimal discrete spheres previously presented.

Proposition 3. Let $\mu \in \mathbb{R}, \mathbf{n} \in \mathbb{R}^{d}$. Let also $p: \mathbb{R}^{d} \longrightarrow \mathbb{R}$ be a map such that: $\forall \mathbf{x} \in \mathbb{R}^{d}, p(\mathbf{x})=\mathbf{n} \cdot \mathbf{x}+\mu$. The $k$-minimal arithmetic discrete hyperplane $\mathrm{P}\left(\mathbf{n}, \mu, \omega_{k}\right)$ of normal vector $\mathbf{n}$ and translation parameter $\mu$ is defined as:

$$
\mathrm{P}\left(\mathbf{n}, \mu, \omega_{k}\right)=\mathrm{P}\left(\mathbf{n}, \mu, 0, \omega_{(1, k)}\right)=\left\{\mathbf{v} \in \mathbb{Z}^{d} \mid 0 \leq p(\mathbf{v})<\right] \Delta_{1} p(\mathbf{v})\left[_{k}\right\} .
$$




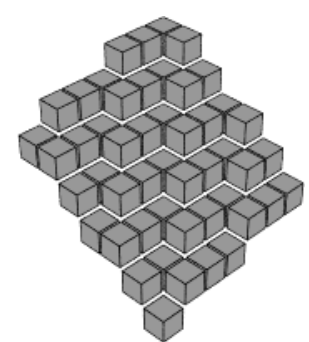

(a) 0-minimal discrete plane

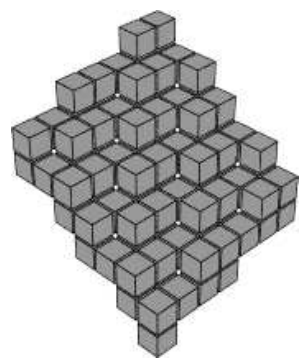

(b) 1-minimal discrete plane

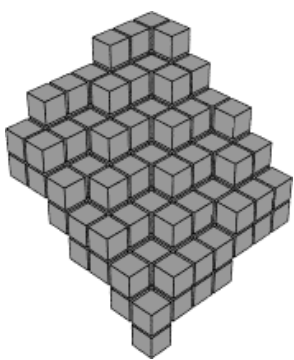

(c) 2-minimal discrete plane

Fig. 3. $k$-minimal discrete planes with normal vector $\mathbf{n}=(1,2,3)$

Consequently, our characterization is not specific to hypersphere but rather a generalization of what we already known about the discrete hyperplane.

\section{Conclusion and Further Research}

In the present paper, we investigated the discrete hypersphere in order to provide it with a definition related to thickness and verifying basic topological properties. The first result is a general arithmetic definition of the discrete hypersphere. In this definition, usual constant arithmetic thickness is replaced by two thickness functions in order to take into account the non-linearity of hyperspheres and to distribute global thickness more or less inside or outside the hypersphere. By relating the $k$-adjacency and the $k$-minimality to norms we control precisely the thickness for discrete sets with non linear analytical description. We thus based thickness functions on the measurement by the k-minimality norm of a vector related to the local behavior of the hypersphere and characterized the $\mathrm{k}$-minimal arithmetic discrete hypersphere.

This characterization could be helpful to infer drawing and recognition algorithms. In the case of the discrete line, the arithmetic definition has improved the understanding of such algorithms. Why the same situation does not apply to the hypersphere or at least to the circle?

Moreover, beyond the particular case of the hypersphere, we go further in the understanding of the arithmetic discrete geometry. Focusing on simple objects, namely lines or circles, reduces the number of parameters in play and allows to better study each of them. Two points seem to be essential and common to all objects arithmetically studied: the use of norms and the study of the local discrete behaviour of the object. As far as we know, remarkable arithmetic discrete objects have always been characterized by measuring, with norms, their local variations. Lines or hyperplanes are linear objects and their normal vector is constant in magnitude and direction. For circles and hyperspheres, the normal vector is only constant in magnitude. The next natural step would be the arithmetic study of hypersurfaces for which the normal vector would have neither a 
constant magnitude nor a constant direction, in other words, the general case of hypersurfaces based on polynomials.

\section{References}

1. Bresenham, J.: Algorithm for computer control of a digital plotter. IBM Systems Journal 4(1) (1965) 25-30

2. Freeman, H.: Computer processing of line-drawing images. ACM Computing Surveys 6(1) (1974) 57-97

3. Rosenfeld, A.: Digital straight lines segments. In: IEEE Transactions on Computers. (1974) 1264-1369

4. Reveillès, J.P.: Géométrie discrète, calcul en nombres entiers et algorithmique. Thèse d'Etat, Université Louis Pasteur, Strasbourg (1991)

5. Debled-Rennesson, I., Reveillès, J.P.: A linear algorithm for segmentation of digital curves. IJPRAI 9(4) (1995) 635-662

6. Andres, E., Acharya, R., Sibata, C.: Discrete analytical hyperplanes. CVGIP: Graphical Models and Image Processing 59(5) (1997) 302-309

7. Bresenham, J.: A linear algorithm for incremental digital display of circular arcs. Communication of the ACM 20(2) (1977) 100-106

8. McIlroy, M.: Best approximate circles on integer grids. ACM Transactions on Graphics 2(4) (1983) 237-263

9. Pham, S.: Digital circles with non-lattice point centers. The Visual Computer 9(1) (1992) 1-24

10. Andres, E., Jacob, M.A.: The discrete analytical hyperspheres. IEEE Transactions on Visualization and Computer Graphics 3(1) (1997) 75-86

11. Fiorio, C., Jamet, D., Toutant, J.L.: Discrete circles: an arithmetical approach with non-constant thickness. In: Vision Geometry XIV, Electronic Imaging, SPIE, San Jose (CA), USA (2006) 